\title{
Do significant TFE3 gene rearrangements occur in succinate dehydrogenase-deficient renal cell carcinoma? Borderline FISH results should be interpreted with caution
}

\author{
Modern Pathology (2017) 30, 1507-1508; doi:10.1038/modpathol.2017.77
}

To the Editor: We read with great interest the recent report by Calio et $a^{1}$ suggesting the presence of TFE3 gene rearrangements in four succinate dehydrogenase (SDH)-deficient renal carcinomas, all of which demonstrated negative staining for SDHB and three of which were associated with confirmed $S D H B$ gene mutation. We were surprised by this finding as we previously considered the presence of TFE3 translocations and $S D H$ mutations as mutually exclusive. Indeed they are considered the defining molecular event of different tumor types in the recent World Health Organization classification of renal neoplasia. ${ }^{2}$ As both may occur in younger patients, our experience has been that some cases of SDHdeficient renal cell carcinoma are only diagnosed after TFE3 gene rearrangements have been excluded. In fact we have never previously encountered both molecular abnormalities in the same tumour. We therefore sought to independently test this finding by searching for TFE3 gene rearrangements using fluorescence in-situ hybridization (FISH) in a large series of SDH-deficient renal cell carcinomas.

Our cohort comprised 30 confirmed SDH-deficient renal cell carcinomas (17 of which have been previously reported $)^{3}$ from 26 patients. By immunohistochemistry five tumors showed focal weak to moderate TFE3 nuclear staining of much lower intensity than found in external positive controls. The remaining 25 tumors were completely negative for TFE3 by immunohistochemistry. Analysis for TFE3 rearrangements using a break-apart FISH assay (Zytovision probe) was performed on all 30 tumors. FISH was interpreted using the same scoring criteria requiring $\geq 10 \%$ tumor nuclei to demonstrate the split-signal pattern to be indicative of gene rearrangement. ${ }^{4,5}$

In our cohort the percentage of split-signal patterns ranged from 0 to $8 \%$ (mean $4.5 \%$ ). That is, using these scoring criteria there were no TFE3 rearrangements in any of $30 \mathrm{SDH}$-deficient renal carcinomas we tested.

What are the possible explanations for the differences in the FISH results observed in our study and those observed by Calio et al? We note that the authors found the percentage of TFE3 split-signal patterns in their cohort was quite low, ranging from 12 to $19 \%$ (mean 16\%). This is significantly lower than their test validation with positive controls $(n=18)$ from patients with confirmed Xp11.2 translocated tumours, which demonstrated $17-78 \%$ split signals (mean $33 \%$ ), but greater than the percentage of split signals in their negative control cohort of clear cell carcinomas (range $0-7 \%$, mean $2 \%$ ). ${ }^{1}$ Others investigating the use of TFE3 FISH diagnostically have also found a high frequency of TFE3 split signal patterns in confirmed Xp11.2 translocated carcinomas (mean $=64 \%$; range $33-94 \%)^{5}$

Current FISH guidelines recommend that borderline-positive and borderline-negative results should always be interpreted with great caution and in the context of other clinical and laboratory findings, and we would consider the authors' FISH results as borderline. 6 Therefore we would suggest that the significance of the authors' finding of lowfrequency TFE3 split signal patterns should be interpreted with caution. Of course, as the authors suggest, perhaps TFE3 gene rearrangements could occur as a late event in small proportions of malignant cells in SDH-deficient renal carcinoma. However, the fact that we found no positive cases in our series of $30 \mathrm{SDH}$-deficient renal carcinomas suggests that this may not be the case, or rather that if it does occur as a secondary event then it occurs no more frequently than in other types of renal cell carcinoma.

For these reasons we think that based on current data TFE3 gene rearrangements are unlikely to be a significant event in SDH-deficient renal carcinoma. Ultimately, further studies may be required to resolve this issue with certainty, but until then we would consider this association unproven.

\section{Disclosure/conflict of interest}

The authors declare no conflict of interest.

Angela Chou ${ }^{1,2,3}$, Ondrej Hes ${ }^{4}$, John Turchini ${ }^{3,4,5}$, Kiril Trpkov ${ }^{6}$ and Anthony J Gill ${ }^{3,4,5}$

${ }^{1}$ Department of Anatomical Pathology, SYDPATH St Vincent's Hospital, Darlinghurst, NSW, Australia;

${ }^{2}$ The Kinghorn Cancer Centre and Garvan Institute of Medical Research, Darlinghurst, NSW, Australia; ${ }^{3}$ Cancer Diagnosis and Pathology Research Group, Kolling Institute of Medical Research, University of Sydney, Royal North Shore Hospital, St Leonards, NSW, Australia; ${ }^{4}$ Department of Pathology, Medical 
Faculty and Charles University, Pilsen, Czech Republic; ${ }^{5}$ Department of Anatomical Pathology, Royal North Shore Hospital, St Leonards, NSW, Australia; ${ }^{6}$ Department of Pathology and Laboratory Medicine, Calgary Laboratory Services and University of Calgary, Calgary, AB, Canada E-mail: affgill@med.usyd.edu.au

\section{References}

1 Calio A, Grignon DJ, Stohr BA, et al. Renal cell carcinoma with TFE3 translocation and succinate dehydrogenase B mutation. Mod Pathol 2017;30:407-415.

2 Moch H, Cubilla AL, Humphrey PA, et al. The 2016 WHO classification of tumours of the urinary system and male genital organs-Part A: renal, penile, and testicular tumours. Eur Urol 2016;70:93-105.
3 Gill AJ, Hes O, Papathomas T, et al. Succinate dehydrogenase (SDH)-deficient renal carcinoma: a morphologically distinct entity: a clinicopathologic series of 36 tumors from 27 patients. Am J Surg Pathol 2014;38: 1588-1602.

4 Rao Q, Williamson SR, Zhang S, et al. TFE3 break-apart FISH has a higher sensitivity for Xp11.2 translocationassociated renal cell carcinoma compared with TFE3 or cathepsin $\mathrm{K}$ immunohistochemical staining alone: expanding the morphologic spectrum. Am J Surg Pathol 2013;37:804-815.

5 Green WM, Yonescu R, Morsberger L, et al. Utilization of a TFE3 break-apart FISH assay in a renal tumor consultation service. Am J Surg Pathol 2013;37: 1150-1163.

6 Mascarello JT, Hirsch B, Kearney HM, et al. Section E9 of the American College of Medical Genetics technical standards and guidelines: fluorescence in situ hybridization. Genet Med 2011;13:667-675. 